\title{
Erythropoietin Enhances Hippocampal Response during Memory Retrieval in Humans
}

\author{
Kamilla Miskowiak, ${ }^{1,2}$ Ursula O'Sullivan, ${ }^{2}$ and Catherine J. Harmer ${ }^{1,2}$ \\ ${ }^{1}$ Department of Experimental Psychology, University of Oxford, Oxford OX1 3UD, United Kingdom, and 2Department of Psychiatry, University of Oxford, \\ Warneford Hospital, Oxford OX3 7JX, United Kingdom
}

\begin{abstract}
Although erythropoietin (Epo) is best known for its effects on erythropoiesis, recent evidence suggests that it also has neurotrophic and neuroprotective properties in animal models of hippocampal function. Such an action in humans would make it an intriguing novel compound for the treatment of neurological and psychiatric disorders. The current study therefore aimed to explore the effects of Epo on neural and behavioral measures of hippocampal function in humans using a functional magnetic resonance imaging paradigm. Volunteers were randomized to receive intravenous injection of Epo (40,000 IU) or saline in a between-subjects, double-blind, randomized design. Neural response during picture encoding and retrieval was tested 1 week later. Epo increased hippocampus response during picture retrieval $(n=11)$ compared with placebo $(n=12 ; p=0.04)$ independent of changes in hematocrit. This is consistent with upregulation of hippocampal BDNF and neurotrophic actions found in animals and highlights Epo as a promising candidate for treatment of psychiatric disorders.
\end{abstract}

Key words: erythropoietin; hippocampal function; cognitive; fMRI; human; neurogenesis

\section{Introduction}

It is now well established that neurogenesis occurs in the adult brain and is confined to the dentate gyrus (DG) of the hippocampus in humans (Lledo et al., 2006). Adult neurogenesis is believed to play a key role in learning and memory and adaptation to novel circumstances (Chambers et al., 2004). Stress-induced elevation of glucocorticoids has been reported to decrease hippocampal neurogenesis and cause spatial learning deficits (Kitraki et al., 2004) and depression-like behavior in rodents (Malberg and Duman, 2003). In humans, persistent cortisol elevation is associated with hippocampal volume reduction and impairment of hippocampus-dependent memory (Lupien et al., 1998), which is thought to be involved in the pathophysiology of depression (Campbell and Macqueen, 2004). Novel compounds that facilitate hippocampal plasticity and neurogenesis may therefore have important implications for the treatment of neurological and neuropsychiatric disorders.

Erythropoietin (Epo) is a natural body-own protein originally identified for its role in erythropoiesis (Jacobson et al., 1957) and used successfully in the treatment of anemia for the past two decades. In addition, it was recently discovered that Epo and its receptor (Epo-R) are expressed throughout the brain in glial cells, neurons, and endothelial cells exerting vital neurobiological actions (Brines and Cerami, 2005). Systemically administered Epo crosses the blood-brain barrier (BBB) (Brines et al., 2000; Ehren-

Received Nov. 18, 2006; revised Jan. 15, 2007; accepted Jan. 30, 2007.

This work was supported by the Lundbeckfonden, Denmark, the Economic and Social Research Council, UK, and the Goodger Scholarship, UK.

Correspondence should be addressed to Kamilla Miskowiak, Department of Psychiatry, University of Oxford, Warneford Hospital, 0xford 0X3 7JX, UK. E-mail: kamilla.miskowiak@psy.ox.ac.uk.

DOI:10.1523/JNEUROSCI.5013-06.2007

Copyright $\odot 2007$ Society for Neuroscience $\quad$ 0270-6474/07/272788-05\$15.00/0 reich et al., 2004) and has potent neuroprotective and neurotrophic effects in traumatic, hypoxic-ischemic, excitotoxic, and inflammatory brain damage in animal models (Morishita et al., 1997; Sakanaka et al., 1998; Brines et al., 2000) accompanied by improved functional recovery (Mogensen et al., 2004). Several mechanisms mediate the neuroprotective actions, including rapid upregulation of biologically active BDNF (Viviani et al., 2005) and neurogenesis (Brines and Cerami, 2005).

Despite growing evidence for the clinical potential of Epo in human neurological and psychiatric disorder, the vast majority of studies have used animal models, and it is unknown whether Epo has similar effects on hippocampal function in humans. A recent study of the effects of Epo on cognitive function in humans found that weekly administration of Epo (40,000 IU) versus saline to schizophrenic patients improved executive function and memory after 12 weeks (Ehrenreich et al., 2007). However, interpretation of these effects as representing direct neuroprotective actions of Epo is confounded by increased red cell mass in patients given Epo versus placebo. Hence, we aimed to explore the effects of acute administration of Epo, which has limited effects on red cell mass, while maintaining effects on neurobiological function (Ehrenreich et al., 2002).

The objective of the current study was to explore the effects of Epo on hippocampal function in humans using functional magnetic resonance imaging (fMRI). Evidence that abnormal intracellular trafficking and secretion of BDNF is associated with reduced hippocampal response and impaired memory during picture encoding and recognition (Hariri et al., 2003) and the finding that Epo increases hippocampal BDNF led us to hypothesize greater memory-related hippocampus response in participants treated with Epo compared with placebo. 


\section{Materials and Methods}

Subjects. Ethical approval for the study was obtained from the Oxfordshire Research Ethics Committee. Healthy subjects were screened through a medical examination and psychiatric interview using the Structured Clinical Interview for DSM-Clinical Version (SCID-IV). Exclusion criteria were as follows: history of psychiatric disorder, significant medical conditions (including diabetes, epilepsy, hypertension, and thrombosis), previous exposure to recombinant human erythropoietin (rh-EPO), pregnancy, current medication (including the contraceptive pill), and first-degree family history of blood clotting or seizure disorders. fMRI scanning also required the following exclusion criteria: cardiac pacemaker, mechanical heart valve, or any mechanical implants, potential pregnancy, and claustrophobia. A baseline venepuncture was done to check that hematocrit, hemoglobin $(\mathrm{Hb})$, renal function, liver function, and ferritin levels were normal, and a pregnancy test was performed on female volunteers. After a complete description of the study to the subjects, written informed consent was obtained.

Experimental design. Twenty-four healthy volunteers were randomly allocated to receive an intravenous injection of either Epo (40,000 IU) or placebo in a double-blind between-groups design. The two groups were matched for the following: gender (8 males per group), age (mean \pm SD: $27.7 \pm 7.9$ and $26.3 \pm 5.0$ ), and IQ measured with the National Adult Reading Test (test score, mean \pm SD, $116.8 \pm 4.9$ and $113.4 \pm 6.2$ ). Blood pressure, well being, and subjective state were monitored for $3 \mathrm{~h}$ after the injection. On day $7 / 8$, we assessed the neural response during picture encoding and recognition. A follow-up blood test was taken to check for any rise in levels of hematocrit, $\mathrm{Hb}$, and red cell count in the experimental group.

Picture encoding and recognition. Stimuli pictures were projected from a computer using e-prime software (version 1.0; Psychology Software Tools, Pittsburgh, PA) onto an opaque screen at the foot of the scanner bore. Subjects viewed the screen through angled mirrors and responded by pressing the keys of a response pad with their thumbs. Thereby, accuracy and reaction time could be investigated.

Picture encoding and recognition tasks were selected because of hippocampal engagement in encoding and retrieval of complex visual scenes (Stern et al., 1996; Hariri et al., 2003) and modified from Hariri et al. (2003). Stimuli pictures were matched for emotional valence, arousal, and visual complexity and were presented in a blocked paradigm to maximize sensitivity for hippocampal blood oxygenation leveldependent (BOLD) signal change (Birn et al., 2002). In both tasks, each of the eight picture blocks ( $24 \mathrm{~s}$ ) were preceded by brief instructions ( $2 \mathrm{~s}$ ) and presented interleaved with $20 \mathrm{~s}$ of fixation cross, resulting in a task duration of $6 \mathrm{~min} 2 \mathrm{~s}$. Blocks consisted of 6 pictures presented serially for $3 \mathrm{~s}$ interleaved with a $1 \mathrm{~s}$ fixation cross. Encoding and recognition tasks contained an equal number of pictures representing indoor and outdoor scenes, and during the recognition part, there was an equal number of old (i.e., previously encoded) and new pictures. During encoding, volunteers determined whether the picture represented an "indoor" or "outdoor" scene, and in the subsequent recognition task, whether the picture was "old" or "new."

Visual stimulation paradigm. To explore whether any drug-related effects observed during picture retrieval were attributable to global effects of Epo on baseline blood flow or neuronal coupling, neural activation was assessed with a visual stimulation paradigm. A flashing checkerboard (frequency, $8 \mathrm{~Hz}$ ) was presented in blocks of $15 \mathrm{~s}$ alternating with $15 \mathrm{~s}$ of a fixation cross for a total of 20 cycles. Subjects were instructed to lie with their eyes open during this control task.

fMRI data acquisition. Imaging data were collected using a Siemens (Erlangen, Germany) 1.5T Sonata scanner at the Oxford Centre for Clinical Magnetic Resonance Research. Functional imaging consisted of 35 T2*-weighted echo-planar image (EPI) slices [repetition time (TR), 3000 ms; echo time (TE), $54 \mathrm{~ms}$; matrix, $128 \times 128], 1.5 \times 1.5 \times 4.5 \mathrm{~mm}^{3}$ voxels. To facilitate later coregistration of the fMRI data into standard space, we also acquired a Turbo FLASH sequence (TR, 12 ms; TE, 5.65 $\mathrm{ms}$ ), voxel size of $1 \mathrm{~mm}^{3}$. The first two EPIs in each session were discarded to avoid T1 equilibration effects.

$f M R I$ data analysis. One subject was excluded from the fMRI analysis because of left-handedness. The analysis was subsequently run on 23 subjects, of whom 11 had been given Epo. fMRI data were preprocessed and analyzed using FEAT (FMRI Expert Analysis Tool) version 5.43, part of Functional Magnetic Resonance Imaging of the Brain (FMRIB) Software Library version $3.2 \beta$ (www.fmrib.ox.ac.uk/fsl). This included within-subject image realignment (Jenkinson et al., 2002), nonbrain removal, spatial normalization to a standard template (Montreal Neurological Institute 152 stereotactic template) using an affine procedure (Jenkinson and Smith, 2001), and spatial smoothing using a Gaussian kernel ( $5 \mathrm{~mm}$ full-width half-maximum). The time series in each session was high-pass filtered (Gaussian-weighted least-squares fit straight line fitting, with $\sigma=40 \mathrm{~s}$ ) to remove large-scale nonstationary components and low-frequency noise. FILM (FMRIB's Improved Linear Model) was used to analyze individual subject data in a general linear model in which the time series were prewhitened to remove temporal autocorrelation. In these analyses, temporal derivatives of the principal regressors were included as covariates of no interest to provide temporal flexibility in model fitting. A mixed-effects group cluster analysis (second-level analysis) was carried out to establish group effects for the stimuli contrasts (higher-level GLM analysis; cluster corrected for multiple comparisons; $z=2.0 ; p=0.05$ ). Brain areas with significant activation were localized using Talairach coordinates (Talairach and Tournoux, 1988).

To investigate responses specific to the hippocampus during picture encoding and recognition, a region of interest (ROI) analysis was performed. ROIs for the left and right hippocampus in standard space were obtained using mri3dX (http://www.aston.ac.uk/lhs/staff/singhkd/ $\mathrm{mri3dX} / \mathrm{mri3dX}$.jsp), which utilizes a stored representation of the Talairach Daemon Database (Lancaster et al., 2000). Mean percentage hippocampal BOLD signal change during encoding and recognition was computed and compared between Epo and placebo groups.

For the control stimulation paradigm, we compared mean percentage BOLD signal change in subjects given Epo versus placebo in a region of the occipital (calcarine) cortex activated by photic stimuli. The mask was based on standardized neuroanatomical divisions (Maldjian et al., 2003). Any effect of Epo in this region would suggest a global drug effect on baseline cerebral hemodynamics or neural coupling.

Statistical analysis of behavioral and mood data. Behavioral data were analyzed using repeated-measures ANOVA with group as the betweensubjects factor and time as the within-subjects factor. Significant interactions were analyzed further using simple main effect analyses. To obtain an additional measure of memory accuracy corrected for the subject's response tendency, signal detection theory was applied. The proportion of correctly recognized pictures (cr) and of falsely recognized pictures (fr) constitute the parametric sensitivity measure: $d^{\prime}=0.5((\mathrm{cr}-$ fr) $(1+\mathrm{cr}-\mathrm{fr}) / 4 \mathrm{cr}(1-\mathrm{fr}))$, with a higher $d$-value reflecting greater accuracy of memory (Grier, 1971). Statistical analyses were performed using the Statistical Package for Social Sciences (SPSS, Chicago, IL).

\section{Results}

It has been hypothesized that beneficial effects of Epo on cognitive function in anemic patients result from increased level of red blood cells and hence better oxygen delivery to the brain. To assess whether such indirect effects could confound the interpretation of our data, we collected blood samples before and after Epo/saline administration. Consistent with our prediction of a direct neurobiological action of Epo on the human CNS, we found no differences between the groups in hemoglobin, hematocrit, or red cell count (ANOVA; all $p>0.31$ ) (Fig. 1).

\section{Neural response during picture encoding and recognition}

As expected, left and right hippocampus were significantly activated across all subjects during picture encoding (ANOVA; main effect of encoding, $\left.F_{(1,22)}=35.5, p<0.001\right)$ and recognition (ANOVA; main effect of recognition, $F_{(1,22)}=21.6, p<0.001$ ). Specifically, Epo significantly enhanced bilateral hippocampus response during picture recognition compared with placebo (main effect of group, $F_{(1,21)}=4.7, p=0.04$ ) (Fig. 2). 


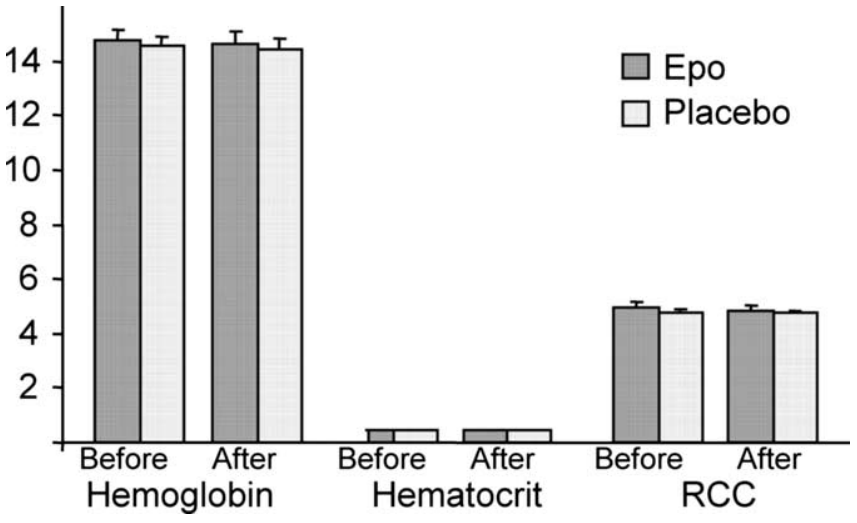

Figure 1. Mean levels of $\mathrm{Hb}$, hematocrit, and red cell count (RCC) before and after administration of Epo $(n=12)$ or placebo $(n=12)$. There was no significant effect of Epo on $\mathrm{Hb}$ $\left(F_{(1,22)}=0.00 ; p=0.98\right)$, hematocrit $\left(F_{(1,22)}=1.10 ; p=0.31\right)$, or $\operatorname{RCC}\left(F_{(1,21)}=0.29 ; p=\right.$ $0.60)$. Error bars represent the SEM.

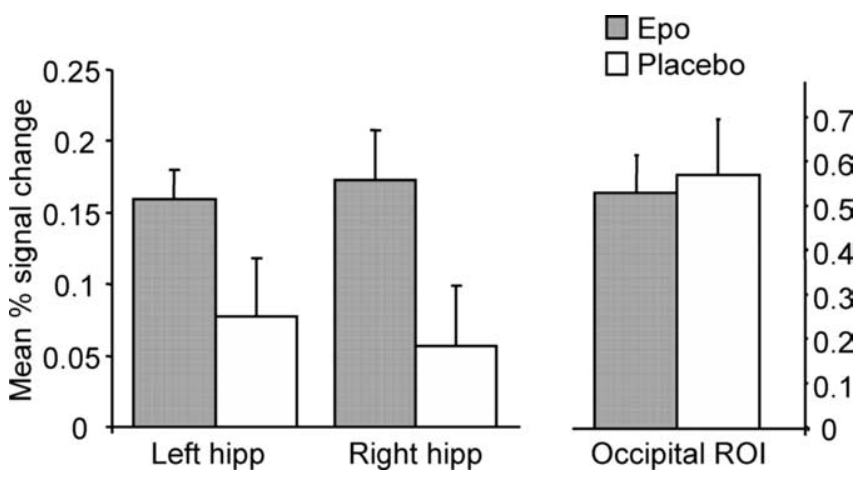

Figure 2. BOLD signal change in the hippocampus (hipp) during picture recognition and in the occipital ROI during photic stimulation. Epo $(n=11)$ enhanced bilateral hippocampal BOLD signal change during picture recognition compared with placebo ( $n=12$; ANOVA; main effect of group, $F_{(1,21)}=4.7, p=0.04$ ) independent of effects on occipital signal change within the visual ROI $(p>0.8)$. Values represent mean percentage signal change. Error bars represent the SEM.

To explore whether Epo influenced neural response in other brain regions, we performed whole-brain mixed-effects group cluster analyses (thresholded at $z=2.0, p=0.05$, corrected). This revealed that Epo influenced a broad neuronal network engaged in encoding and recognition of complex visual scenes (for cluster maxima, see Table 1). During encoding, Epo-treated volunteers displayed increased activation in a left-lateralized frontoparietal network including the middle frontal gyrus, caudate, precuneus, and cuneus (Fig. $3 a$; for cluster maxima, see Table 1). During recognition, Epo upregulated activation in the bilateral superior frontal cortex and left middle and inferior temporal cortices (Fig. $3 b$; for cluster maxima, see Table 1 ).

\section{Memory performance}

Recognition accuracy during the encoding and recognition tasks were high (mean encoding accuracy, 98\%; mean recognition accuracy corrected for response bias, $\left.d^{\prime}=0.85\right)$. Epo did not affect accuracy or response times in either of the tasks (all $p>0.05$ ).

\section{Visual stimulation paradigm}

A region of occipital (calcarine) cortex activated by photic stimulation across all subjects was identified as an ROI. Analysis of mean percentage BOLD signal change in this region revealed no differences between groups $(p>0.8)$ (Fig. 2), indicating that the observed effects of Epo did not result from global hemodynamic changes.

\section{Discussion}

The present study is the first to investigate the actions of Epo on neurobiological and cognitive function in humans using functional neuroimaging. We demonstrated that one dose of Epo versus saline modified the neural underpinnings of picture encoding and recognition in healthy volunteers. In particular, Epo enhanced bilateral hippocampal response during picture recognition 1 week after administration. The absence of changes in hematological parameters indicates that our findings reflect direct neurobiological actions of Epo.

The enhanced bilateral hippocampal response in Epo-treated volunteers is consistent with evidence that Epo upregulates biologically active hippocampal BDNF (Viviani et al., 2005) and produces effects opposite to those associated with abnormal BDNF trafficking in a similar memory task (Hariri et al., 2003). Increased hippocampal activation has been reported during correct versus incorrect picture recognition (Cansino et al., 2002), which is compatible with a beneficial effect of Epo on recognition memory.

The DG of the hippocampus plays a role in spatial memory acquisition, which suggests that neurogenesis in this region is important for spatial learning and memory. Newborn cells take weeks to mature but may influence learning processes in early stages because of unique properties such as increased capacity for long-term potentiation, a main neurobiological mechanism underlying early memory formation (Aimone et al., 2006). Computational simulation shows that neurogenesis improves network capacity for new information storage and forgetting of old irrelevant information (Chambers et al., 2004). Increased hippocampal neurogenesis may hence improve learning and adaptive cognitive and emotional responses to novel challenging contexts, whereas reduced neurogenesis might impair ability to cope with stress and be a basis for psychiatric disorders like depression (Chambers et al., 2004). If upregulation of BDNF-mediated neuroplasticity and neurogenesis in the hippocampus and increased response in hippocampus-dependent tasks are important factors in the treatment of depression as hypothesized, Epo seems a promising candidate for novel treatment strategies.

Whole-brain exploratory analysis revealed that Epo increased activation in a left-lateralized frontoparietal network during picture encoding. Enhanced frontoparietal activation during picture encoding predicts retrieval success (Cansino et al., 2002; Iidaka et al., 2006), in agreement with our hypothesis of augmented memory processes after Epo administration. During recognition, Epo additionally upregulated activation in the bilateral superior frontal cortex and temporal cortices, again consistent with enhanced recognition memory (Cansino et al., 2002). Human adult neurogenesis has only consistently been demonstrated in the DG (Lledo et al., 2006) and is unlikely to be present in neocortical regions (Bhardwaj et al., 2006). It is possible that the effects of Epo on activation in the frontoparietal network are modulated indirectly through the increased hippocampal response during retrieval memory. However, according to the whole-brain analysis, the effects of Epo on response in these regions were stronger than the hippocampal effect. In light of this, it is perhaps more likely that these effects represent increased BDNF-mediated neuroplasticity within several cortical regions rather than increased hippocampal neurogenesis per se.

Despite the beneficial effects of Epo on neural response during retrieval memory, we found no effects on recognition perfor- 
Table 1. Brain regions showing significant activation during picture encoding and recognition and effects of Epo $(n=11)$ versus placebo ( $n=12$; thresholded at $z=2.0$ and $p<0.05$, corrected)

\begin{tabular}{|c|c|c|c|c|}
\hline \multirow[b]{2}{*}{ Task and region } & \multirow[b]{2}{*}{$z$-value } & \multicolumn{3}{|c|}{ Coordinates } \\
\hline & & $x$ & $y$ & $z$ \\
\hline \multicolumn{5}{|l|}{ Picture encoding } \\
\hline \multicolumn{5}{|l|}{ Main effect of task } \\
\hline Right calcarine sulcus (BA 17) & 7.18 & 8 & -86 & -4 \\
\hline Left anterior cingulate gyrus (BA 32) & 4.45 & -4 & 2 & 54 \\
\hline Right inferior frontal gyrus (BA 44) & 5.64 & 48 & 6 & 32 \\
\hline \multicolumn{5}{|l|}{ Epo $>$ Placebo } \\
\hline Left precuneus (BA 19) & 3.38 & -14 & -86 & 40 \\
\hline Left caudate body & 3.53 & -10 & 12 & 18 \\
\hline \multicolumn{5}{|l|}{ Picture recognition } \\
\hline \multicolumn{5}{|l|}{ Main effect of task } \\
\hline Left fusiform gyrus (BA 37) & 8.43 & -36 & -56 & -14 \\
\hline \multicolumn{5}{|l|}{ Epo $>$ Placebo } \\
\hline Left medial frontal gyrus (BA 9) & 3.21 & -8 & 46 & 46 \\
\hline Right superior frontal gyrus (BA 9) & 3.26 & 14 & 48 & 40 \\
\hline Left inferior temporal gyrus (BA 21) & 4.18 & -60 & -8 & -18 \\
\hline
\end{tabular}

MNI coordinates $(x, y, z)$ refer to peak activation within each cluster identified thresholded at $z=2.0$ and $p<0.05$, corrected. BA, Brodmann area. consistent with neurotrophic actions of Epo within the hippocampus, the BOLD measure is not a specific measure of BDNF-mediated neural plasticity per se. Additional research is required to assess whether other effects such as improved cognitive function are also found after Epo administration. Furthermore, animal experiments could help define whether effects of Epo in depression models are abolished after irradiation of the hippocampus, consistent with results seen with conventional antidepressants (Santarelli et al., 2003). However, the results of the present study, coupled with previous findings (Hariri et al., 2003), are certainly consistent with this hypothesis and suggest that additional investigation of the actions of Epo is warranted. Such actions would make Epo an intriguing candidate
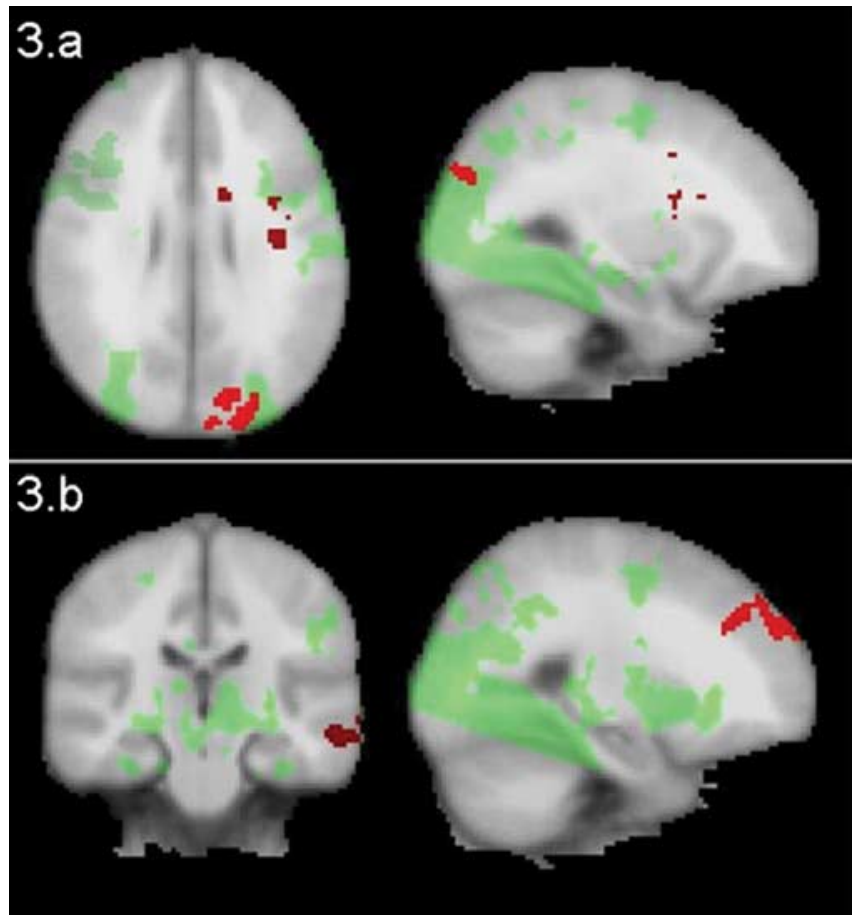

Figure 3. $\boldsymbol{a}$, Neuronal response during picture encoding. Epo $(n=11)$ increased activation during picture encoding in a network encompassing the middle frontal gyrus, caudate, precuneus, and cuneus compared with placebo $(n=12)$. $\boldsymbol{b}$, Neuronal response during picture recognition. Epo $(n=11)$ increased activation in bilateral frontal and the left middle and inferior temporal cortices compared with placebo $(n=12)$. Areas of increased response under Epo versus placebo are marked with red; areas activated by task under placebo are marked with green. Images are thresholded at $z=2.0, p=0.05$, corrected. For cluster maxima, see Table 1 .

mance. This could be a result of ceiling effects attributable to high accuracy in all participants in this task, which was optimized for the detection of hippocampal response rather than behavioral differences. A parallel set of experiments investigating the behavioral effects of Epo on these measures would have strengthened the interpretation of the current fMRI data. Additional studies using more difficult tasks outside a scanning environment are needed to clarify whether the increased hippocampus engagement in picture retrieval reflects improved memory.

It is important to acknowledge that although these results are for the treatment of neurological and neuropsychiatric disorders, including mood and anxiety disorders.

\section{References}

Aimone JB, Wiles J, Gage FG (2006) Potential role for adult neurogenesis in the encoding of time in new memories. Nat Neurosci 9:723-727.

Bhardwaj RD, Curtis MA, Spalding KL, Buchholz BA, Fink D, Bjork-Eriksson T, Nordborg C, Gage FH, Druid H, Eriksson PS, Frisen J (2006) Neocortical neurogenesis in humans is restricted to development. Proc Natl Acad Sci USA 103:12564-12568.

Birn RM, Cox RW, Bandettini PA (2002) Detection versus estimation in event-related fMRI: choosing the optimal stimulus timing. NeuroImage 15:252-264.

Brines M, Cerami A (2005) Emerging biological roles for erythropoietin in the nervous system. Nat Neurosci 6:484-494.

Brines ML, Ghezzi P, Keenan S, Agnello D, de Lanerolle NC, Cerami C, Itri LM, Cerami A (2000) Erythropoietin crosses the blood-brain barrier to protect against experimental brain injury. Proc Natl Acad Sci USA 97:10526-10531.

Campbell S, Macqueen GJ (2004) The role of the hippocampus in the pathophysiology of major depression. J Psychiatry Neurosci 29:417-426.

Cansino S, Maquet P, Dolan RJ, Rugg MD (2002) Brain activity underlying encoding and retrieval of source memory. Cereb Cortex 12:1048-1056.

Chambers RA, Potenza MN, Hoffman RE, Miranker W (2004) Simulated apoptosis/neurogenesis regulates learning and memory capabilities of adaptive neural networks. Neuropsychopharmacology 29:747-758.

Ehrenreich H, Hasselblatt M, Dembowski C, Cepek L, Lewczuk P, Stiefel M, Rustenbeck HH, Breiter N, Jacob S, Knerlich F, Bohn M, Poser W, Ruther E, Kochen M, Gefeller O, Gleiter C, Wessel TC, De Ryck M, Itri L, Prange H, et al. (2002) Erythropoietin therapy for acute stroke is both safe and beneficial. Mol Med 8:495-505.

Ehrenreich H, Degner D, Meller J, Brines M, Behe M, Hasselblatt M, Woldt H, Falkai P, Knerlich F, Jacob S, von Ahsen N, Maier W, Bruck W, Ruther E, Cerami A, Becker W, Siren AL (2004) Erythropoietin: a candidate compound for neuroprotection in schizophrenia. Mol Psychiatry 9:42-54.

Ehrenreich H, Hinze-Selch D, Stawicki S, Aust C, Knolle-Veentjer S, Wilms S, Heinz G, Erdag S, Jahn H, Degner D, Ritzen M, Mohr A, Wagner M, Schneider U, Bohn M, Huber M, Czernik A, Pollmacher T, Maier W, Siren AL, Klosterkotter J, et al. (2007) Improvement of cognitive function in chronic schizophrenic patients by recombinant human erythropoietin. Mol Psychiatry 12:206-220.

Grier JB (1971) Nonparametric indices for sensitivity and bias: computing formulas. Psychol Bull 75:9424-9429.

Hariri AR, Goldberg TE, Mattay VS, Kolachana BS, Callicott JH, Egan MF, Weinberger DR (2003) Brain-derived neurotrophic factor val66met polymorphism affects human memory-related hippocampal activity and predicts memory performance. J Neurosci 23:6690-6694.

Iidaka T, Matsumoto A, Nogawa J, Yamamoto Y, Sadato N (2006) Frontoparietal network involved in successful retrieval from episodic memory. 
Spatial and temporal analyses using fMRI and ERP. Cereb Cortex $16: 1349-1360$.

Jacobson LO, Goldwasser E, Fried W, Plzak LF (1957) Studies on erythropoiesis. VII. The role of the kidney in the production of Erythropoietin. Trans Assoc Am Physicians 70:305-317.

Jenkinson M, Smith S (2001) A global optimisation method for robust affine registration of brain images. Med Image Anal 5:143-156.

Jenkinson M, Bannister P, Brady M, Smith S (2002) Improved optimization for the robust and accurate linear registration and motion correction of brain images. NeuroImage 17:825-841.

Kitraki E, Kremmyda O, Youlatos D, Alexis M, Kittas C (2004) Spatial performance and corticosteroid receptor status in the 21-day restraint stress paradigm. Ann NY Acad Sci 1018:323-327.

Lancaster JL, Woldorff MG, Parsons LM, Liotti M, Freitas CS, Rainey L, Kochunov PV, Nickerson D, Mikiten SA, Fox PT (2000) Automated Talairach atlas labels for functional brain mapping. Hum Brain Mapp 10:120-131.

Lledo P, Alonso M, Grubb MS (2006) Adult neurogenesis and functional plasticity in neuronal circuits. Nat Neurosci 7:179-193.

Lupien SJ, de Leon M, de Santi S, Convit A, Tarshish C, Nair NP, Thakur M, McEwen BS, Hauger RL, Meaney MJ (1998) Cortisol levels during human aging predict hippocampal atrophy and memory deficits. Nat Neurosci [Erratum (1998) 1:329] 1:69-73.

Malberg JE, Duman RS (2003) Cell proliferation in adult hippocampus is decreased by inescapable stress: reversal by fluoxetine treatment. Neuropsychopharmacology 28:1562-1571.

Maldjian JA, Laurienti PJ, Kraft RA, Burdette JH (2003) An automated method for neuroanatomic and cytoarchitectonic atlas-based interrogation of fMRI data sets. NeuroImage 19:1233-1239.

Mogensen J, Miskowiak K, Sorensen TA, Olsen NV, Springborg JB, Mala H (2004) Erythropoietin improves place learning in fimbria-fornixtransected rats and modifies the search pattern in normal rats. Pharmacol Biochem Behav 77:381-390.

Morishita E, Masuda S, Nagao M, Yasuda Y, Sasaki R (1997) Erythropoietin receptor is expressed in rat hippocampal and cerebral cortical neurons, and erythropoietin prevents in vitro glutamate-induced neuronal death. Neuroscience 76:105-116.

Sakanaka M, Wen TC, Matsuda S, Masuda S, Morishita E, Nagao M, Sasaki R (1998) In vivo evidence that erythropoietin protects neurons from ischemic damage. Proc Natl Acad Sci USA 95:4635-4640.

Santarelli L, Saxe M, Gross C, Surget A, Battaglia F, Dulawa S, Weisstaub N, Lee J, Duman R, Arancio O, Belzung C, Hen R (2003) Requirement of hippocampal neurogenesis for the behavioral effects of antidepressants. Science 301:805-809.

Stern CE, Corkin S, Gonzalez RG, Guimaraes AR, Baker JR, Jennings PJ, Carr CA, Sugiura RM, Vedantham V, Rosen BR (1996) The hippocampal formation participates in novel picture encoding: evidence from functional magnetic resonance imaging. Proc Natl Acad Sci USA 93:8660-8665.

Talairach J, Tournoux P (1988) Co-planar stereotaxic atlas of the human brain. New York: Thieme.

Viviani B, Bartesaghi S, Corsini E, Villa P, Ghezzi P, Garau A, Galli CL, Marinovich M (2005) Erythropoietin protects primary hippocampal neurons increasing the expression of brain-derived neurotrophic factor. J Neurochem 93:412-421. 